\section{Molecular Syndromology}

Mol Syndromol 2017;8:148-154

DOI: $10.1159 / 000467909$
Accepted: January 27, 2017

by M. Schmid

Published online: March 31, 2017

\title{
Novel Marfan Syndrome-Associated Mutation in the FBN1 Gene Caused by Parental Mosaicism and Leading to Abnormal Limb Patterning
}

\author{
Efrén Martínez-Quintana ${ }^{a}$ Noemí Caballero-Sánchez ${ }^{b}$ \\ Fayna Rodríguez-González ${ }^{c}$ Paloma Garay-Sánchez ${ }^{b}$ Antonio Tugores ${ }^{b}$ \\ ${ }^{a}$ Cardiology Service and ${ }^{b}$ Research Unit, Complejo Hospitalario Universitario Insular-Materno Infantil, and \\ 'Ophthamology Service, Hospital Universitario Dr. Negrín, Las Palmas de Gran Canaria, Spain
}

\section{Keywords}

Bone morphogenetic protein · Cysteine · EGF_CA domain . Marfan syndrome $\cdot$ Mosaicism $\cdot$ Patterning

\begin{abstract}
Marfan syndrome is an autosomal dominant disorder of the connective tissue caused by mutations in the fibrillin-1 (FBN1) gene. Mutations affecting cysteine residues within the epidermal growith factor-like calcium-binding domains (EGF_CA) of FBN1 are associated with Marfan syndrome features and, especially, with ectopia lentis. We report a novel substitution, affecting the first cysteine of an EGF_CA-binding module encoded by exon 63 of $F B N 1$ (C2571Y), in a patient presenting with typical Marfan syndrome features but without ectopia lentis. The involvement of this particular carboxi-terminal domain in bone morphogenetic protein signaling is evidenced by patterning defects in the apendicular skeleton shown by the gain of a phalange at digit 1 and the fusion of some wrist bones. Although the mutation appeared as sporadic, detailed analysis revealed that the asymptomatic father was a gonosomal mosaic, and that aproximately $25 \%$ of his body cells carry the mutation. Based on this and previous evidence on the origin of sporadic mutations, we would like to stress the importance of detailed parental genetic screening, so the risk of recurrence may be evaluated.

(c) 2017 S. Karger AG, Basel
\end{abstract}

() 2017 S. Karger AG, Basel
Marfan syndrome (MFS; MIM 154700) is an autosomal dominant disorder of the connective tissue, with a prevalence between 2 and 3 in 10,000, in which cardiovascular, skin, skeletal, ocular, pulmonary, and dura mater abnormalities may be present to a highly variable degree [Judge and Dietz, 2005; Verstraeten et al., 2016]. In most of the cases, MFS is caused by heterozygous mutations in the fibrillin-1 (FBN1) gene, encoding FBN1, a glycoprotein that constitutes the major component of extracellular microfibrils, with a widespread distribution in both elastic and nonelastic connective tissue [Sakai et al., 2016]. FBN1 has not only a structural role, but also participates, along with the closely related latent-transforming growth factor beta (TGF- $\beta$ )-binding proteins, in the signaling machinery of factors belonging to the TGF- $\beta$ superfamily, some of them involved in skin, heart and skeletal development [Sakai et al., 2016; Verstraeten et al., 2016].

There are over 1,800 FBN1 gene mutations [CollodBéroud et al., 2003; Sakai et al., 2016] associated with a broad phenotypic continuum, ranging from isolated features of MFS to a neonatal presentation of a severe and rapidly progressive disease in multiple organ systems [Judge and Dietz, 2005; Verstraeten et al., 2016]. Genotype-phenotype correlations are difficult to interpret due to the pleiotropic nature of the mutations: familial ectopia lentis (MIM 129600), geleophysic dysplasia 2 (MIM

\section{KARGER}

E-Mail karger@karger.com

www.karger.com/msy
Antonio Tugores

Unidad de Investigación, Complejo Hospitalario Universitario Insular-Materno Infantil Avenida Marítima del Sur $\mathrm{s} / \mathrm{n}$

ES-35016 Las Palmas de Gran Canaria (Spain)

E-Mail atugores@yahoo.com 

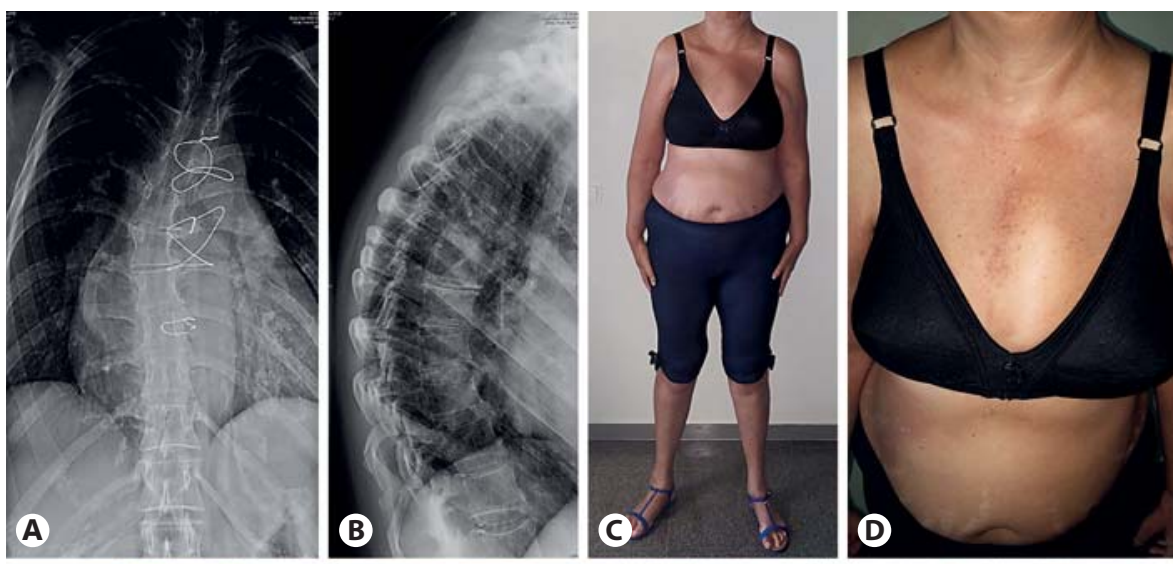

Fig. 1. Clinical features. Dorsal spine X-ray in the anteroposterior $(\mathbf{A})$ and lateral (B) view showing thoracic scoliosis, convex to the right, with spondylosis deformans. Also a normal cardiothoracic ratio and cerclage wires secondary to the patient's cardiac surgery are visible in A. C Anterior total body image of the patient. D Pectus carinatum and abdominal striae atrophicae. E Hands. F High palate.

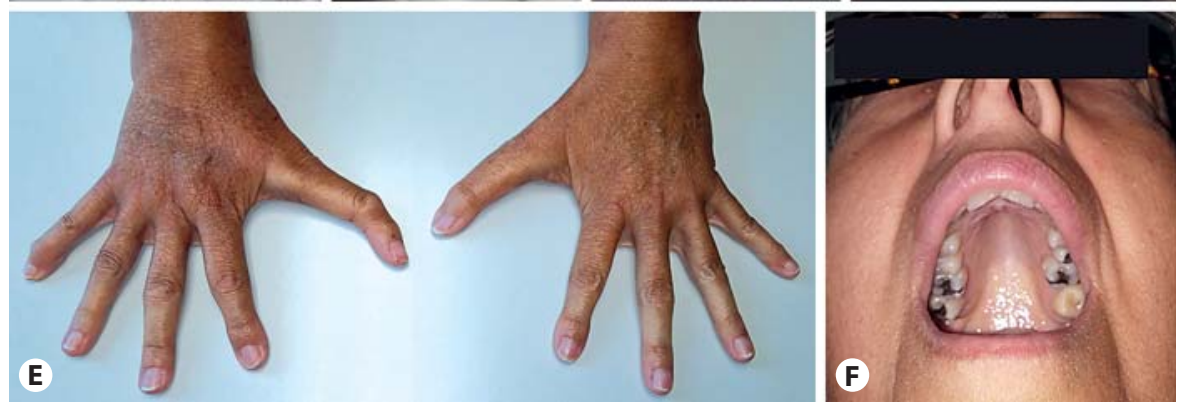

614185), MASS syndrome (mitral valve prolapse, aortic enlargement, skin, and skeletal findings; MIM 604308), stiff skin syndrome (MIM 184900), and dominant WeillMarchesani syndrome 2 (MIM 608328) are distinguishable clinical entities, all caused by FBN1 gene mutations [Sakai et al., 2016].

The FBN1 protein contains a number of repeated structural modules, mostly represented by the epidermal growth factor-like calcium-binding domain (EGF_CA; smart00179) [Sakai et al., 2016]. The functional importance of the 43 EGF_CA modules and their conserved 6 cysteine clusters is evidenced by the high proportion of mutations affecting cysteines within this domain in MFS patients [Dietz et al., 1992; Schrijver et al., 1999; Faivre et al., 2007].

In most cases, mutations are inherited in an autosomal dominant manner, whereas in a large fraction of MFS probands (25-50\%) [Faivre et al., 2007; Sakai et al., 2016; Verstraeten et al., 2016], the disease arises in individuals with no clinical familial history of the disease. One explanation is the occurrence of paternal mosaicism, although it is so rarely reported that it is unlikely to explain all sporadic cases [Montgomery et al., 1998; Collod-Béroud et al., 1999; Tekin et al., 2007; Sípek et al., 2014; Rekondo et al., 2016].

Mosaicism in Sporadic Marfan Syndrome

\section{Methods}

Clinical Analysis

The diagnosis of MFS was done following the revised Ghent criteria [Loeys et al., 2010]. Two-dimensional and pulsed Doppler echocardiography was performed with an iE33 echocardiography system (Philips Medical Systems, Bothell, WA, USA), and aortic dimensions were measured at the sinotubular junction level and at the tubular ascending aorta at $1 \mathrm{~cm}$ above sinotubular junction. $\mathrm{X}$-rays of the chest, dorsal spine, and both hips were performed to evaluate skeletal features. After fasting for at least $10 \mathrm{~h}$, blood samples were drawn for the measurements of serum glucose, serum creatinine, total cholesterol, and hemoglobin.

\section{Genetic Analysis}

After obtaining appropriate informed consent, DNA was extracted from whole venous blood with the Puregene kit (QIAGEN, Hilden, Germany) or from buccal swabs [Walker et al., 1999]. Samples were subjected to double-pass heterozygote Sanger sequencing, covering all exons and flanking sequences of the FBN1 gene. To perform monoallelic sequencing, exon 63 was amplified from DNA extracted from blood by using polymerase chain reaction (PCR) with the oligonucleotide primers FBN1_1F (GGTTCCACTGGTAGTGCTGG) and FBN1_1R (GTAGGATGTGTAGGGGCCAG) for 35 cycles with Phusion High-Fidelity DNA Polymerase (New England Biolabs, Ipswich, MA, USA). The PCR product was then purified (QIAGEN) and subjected to 2 cycles of amplification with Taq DNA polymerase before cloning into the pGEM-T Easy cloning vector and transforming Escherichia coli as recommended by the manufacturer of the kit (Promega Biotech,

Mol Syndromol 2017;8:148-154 DOI: $10.1159 / 000467909$ 
Madison, WI, USA). Individual bacterial clones were sequenced with the FBN1_1F primer. Alignments to similar EGF_CA domains were taken from the Conserved Domain Database [Marchler-Bauer et al., 2015]. The predicted effect of the substitution was evaluated using Polyphen [Sunyaev et al., 2001] and SIFT [Ng and Henikoff, 2003].

\section{Results}

\section{Clinical Evaluation}

A 43-year-old female with no history of MFS or sudden death in her family referred for dyspnea evaluation, with no chest pain or syncope. Cardiac auscultation showed a mild proto-systolic ejection murmur at the pulmonary focus level. Physical examination revealed significant skeletal features: scoliosis, upper/lower segment ratio of 0.73 , arm span/height ratio of 1.1 , pectus carinatum, abdominal striae atrophicae, hands showing arachnodactyly with tall and slender tubular bones and very long proximal phalanges in both thumbs, high palate and dental crowding (Fig. 1) as well as flat feet. Moreover, she presented with myopia and astigmatism, sclerosis of both lenses, and an epimacular nascent membrane in her left eye. No lens dislocation (ectopia lentis) was observed. The X-ray of the spine showed thoracic scoliosis, convex to the right, with spondylosis deformans. Radiography also evidenced a mild left acetabular protrusio at the hip and a wire cerclage of the sternum (related to cardiac surgery) with a normal cardiothoracic ratio (Fig. 1). The right hand had suffered fusion of the carpal bones: the scaphoid to the lunate and, apparently, the trapezoid to the trapezium. Furthermore, the pisiform appeared to be missing or fused to the triquetrum in both hands. The right hand also showed degeneration at the distal interphalangeal joint on digit 5 and an extra phalange on digit 1 (Fig. 2).

A 12-lead electrocardiogram revealed atrial fibrillation. The echocardiogram showed dilatation of the ascending aorta $(40 \mathrm{~mm})$ and the aortic root $(42 \mathrm{~mm})$, high insertion of the papillary muscle with anterior mitral valve prolapse and minimal valve regurgitation, crossing of the chordae tendineae across the left ventricular outflow tract without producing a gradient, and a large ostium secundum atrial septal defect. Laboratory tests were normal.

Percutaneous closure of the atrial septal defect was attempted but was unsuccessful due to its large size (diameter $=35 \mathrm{~mm}$ ) anchoring of a 34-mm Amplatzer septal occluder (AGA Medical Corporation, Golden Valley, MN, USA). During surgery for atrial septal defect closure,
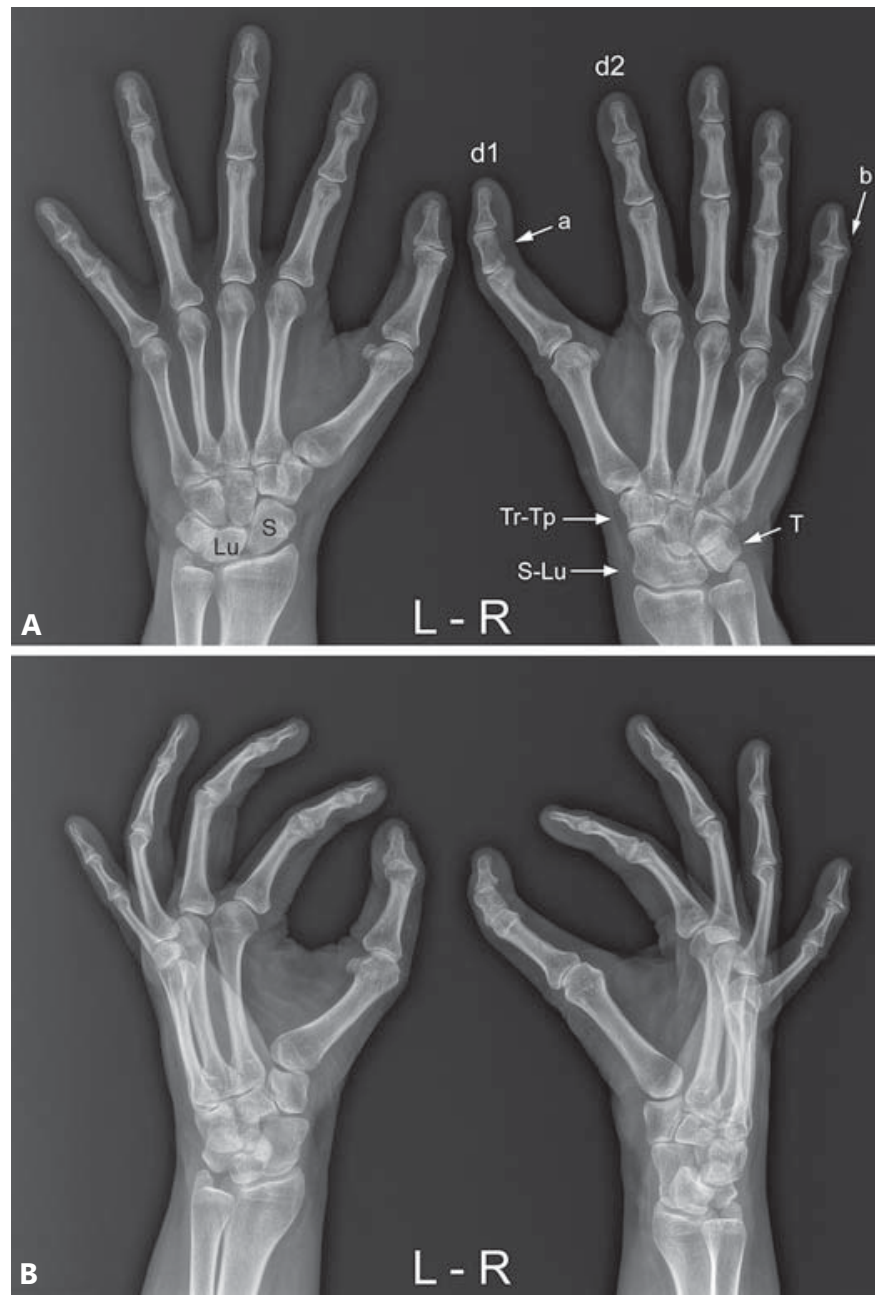

Fig. 2. X-ray analysis of the hands. A Palmar view showing left (L) and right $(\mathrm{R})$ hands as specified. The arrows indicate the extra phalanx on digit 1 of the right hand (a), degenarative changes at the distal interphalangeal region on digit 5 (b), the fusion of the scaphoid (S) and lunate ( $\mathrm{Lu})$ as well as the trapezium (Tr) to the trapezoid (Tp). d1, digit 1; d2, digit 2; T, triquetrum. B Lateral view of both hands.

direct examination of the ascending aorta revealed no evidence of macroscopic alterations except for the dilatation, so it was not resected. Ten years after surgery, the patient is in a I/IV New York Heart Association functional class with no progression in the dilatation of both the aortic root and the ascending aorta.

The father of the patient, examined at age 72, was clinically asymptomatic and had no apparent MFS features. At age 70, presenting with only mild myopia and presbyopia, he underwent cataract surgery. The echocardiogram evidenced a preserved left ventricular function, no 

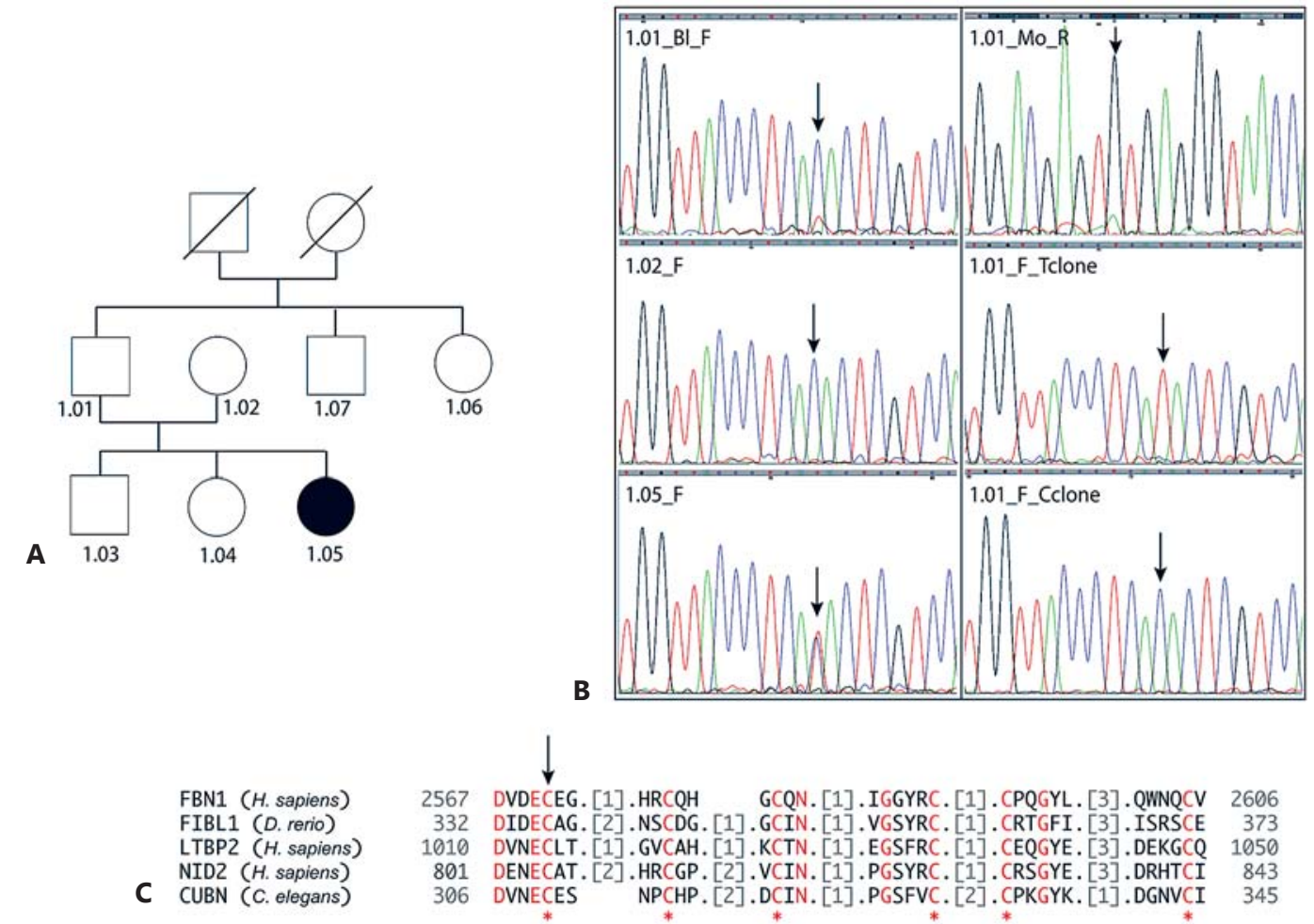

Fig. 3. Genetic analysis. A Family tree showing the situation of the patient 1.05 in relation to other examined relatives (numbered). The individual showing MFS symptoms is shaded. B Chromatograms showing the noncoding strand sequences from DNA isolated from blood cells from the father (1.01_Bl_F), the mother (1.02_F), the affected proband (1.05_F), the sequence of the coding strand from buccal epithelial cells isolated from the father (1.01 Mo_R), and individual clones corresponding to either the wildtype C allele (1.01_F_Cclone) or the mutant T allele (1.01_F_ Tclone) amplified and isolated from the father's DNA. The proband's sequence shows the heterozygous $\mathrm{C}$ to $\mathrm{T}$ nucleotide change in the noncoding strand which leads to a nonsynonymous amino acid substitution Cys to Tyr at position 2,571 of the fibrillin 1 poly- peptide (NP_000129.3). C Primary structure of the epidermal growth factor-like calcium-binding domain (smart00179: EGF_ CA; EGF_CA superfamily accession: cl21504; reference 15). The translated product of exon 63 of the FBN1 gene (NP_000129.3), where the mutation C2571Y is found (arrow), aligned with similar domains from selected examples is shown: FIBL1 corresponds to fibulin-1 from Danio rerio (accession: O42182.1); LTBP2 is the human latent-transforming growth factor beta-binding protein 2 (NP_000419.1);NID2 is the human nidogen-2gene(NP_031387.3), and CUBN corresponds to cubilin from Caenorhabditis elegans (NP_506157.3). The conserved residues are shown in red together with the 6 conserved cysteines (asterisks). valvular pathology, and an ascending aorta of normal size (diameter $=36 \mathrm{~mm}$ ). He was not subjected to X-ray analysis, so there is no detailed skeletal information available.

\section{Genetic Study}

The patient and closest relatives (Fig. 3A) underwent genetic analysis after giving appropriate consent. Sequencing of all exons and flanking sequences of the proband revealed a novel $\mathrm{G}$ to $\mathrm{T}$ substitution at nucleotide 2,571 of the transcript ENST00000316623 (c.8168>T;
chr15:48420794C $>$ T) leading to a nonsynonymous amino acid change (Cys to Tyr) at position 2,571 of FBN1 (NP_000129.3, p.C2571Y). This substitution affects the first of the 6 highly conserved cysteines within the EGF_ CA domain encoded by exon 63 of FBN1 (Fig. 3B). The potential pathogenicity of the variant was evaluated with 2 prediction algorithms, SIFT and Polyphen, that consider the physicochemical consequences of a specific amino acid substitution, as well as the degree of conservation among species, to predict a functional consequence. These algorithms predicted the change to be del- 
eterious (SIFT score $=0$ ) or probably damaging (Polyphen score $=0.999$ ).

Genetic screening within the family revealed that the father had a small $\mathrm{T}$ peak within the $\mathrm{C}$ reference nucleotide (Fig. 3C), suggesting the probability of mosaicism. This finding was repeated in a buccal swab sample and was absent in the mother of the proband (Fig. 3C) as well as 2 of the father's siblings (not shown). To confirm the mosaicism hypothesis, DNA from blood cells was subjected to PCR amplification using a high-fidelity polymerase, and the resulting product was cloned in a prokaryotic vector in order to isolate individual bacterial clones transformed with individual alleles. Sequencing of these clones revealed that $4 / 29(13.8 \%)$ represented the variant allele $(\mathrm{T})$ in the father's DNA (MRF1.01), while it was absent in his sister MRF1.06 (none in 42) and his brother MRF1.07 (none in 26). Both alleles were equally represented in the heterozygous proband, where $6 / 10$ clones represented the variant, demonstrating that there was no allele-specific bias in the cloning procedure.

\section{Discussion}

The EGF_CA domains that comprise most of the FBN1 polypeptide and, in particular, the highly conserved cysteines, host most of the mutations associated with MFS [Dietz et al., 1992; Schrijver et al., 1999; Faivre et al., 2007]. Biochemical evidence shows that these cysteine residues are necessary for proper folding and calcium binding of the EGF_CA module, which in turn participates in proper secretion and deposition of FBN1 [Schrijver et al., 1999].

Consistent with a dominant-negative mechanism of action, there is a great clinical variability. While ectopia lentis seems to be consistently associated with cysteine substitutions, the appearance of both skeletal and cardiovascular phenotypes varies greatly, from the most severe forms of the disease to almost asymptomatic forms [Dietz et al., 1992; Schrijver et al., 1999; Faivre et al., 2007; Martínez-Quintana et al., 2014]. In this context, we report a novel cysteine substitution in a patient presenting with typical MFS features but without ectopia lentis. This substitution affects the first cysteine of an EGF_CA module, participating in a di-sulfide bond whose disruption has been strongly associated with scoliosis [Schrijver et al., 1999; Faivre et al., 2007], as in our patient.

In the absence of a functional test, the potential functional significance of the novel C2571Y variant was evaluated with prediction algorithms that agreed with the phenotype. Interestingly, another patient with a different sporadic mutation at this residue (C2571R) has been identified. Although the skeletal clinical picture appears similar to our patient, ectopia lentis is present in this case, while it was absent in ours [Arbustini et al., 2005].

In addition, the patient presented showed limb patterning defects: the right hand showed fused carpal bones and an extra phalange on digit one. This finding strongly links this particular domain of FBN1 to bone morphogenetic protein (BMP) signaling. The formation of a vertebrate limb arises from the interplay of 2 signaling centers, the apical ectodermal ridge (AER) and the zone of polarizing activity (ZPA), in a canvas orchestrated by Homeobox containing (Hox) genes. The AER, by delivering fibroblast growth factors, stimulates the proliferation of the underlying mesoderm to grow the limb in the proximodistal axis. Simultaneously, the ZPA delivers sonic hedgehog, a signaling molecule that prevents the differentiation of nearby mesoderm cells, ensuring that posterior structures closer to the ZPA are determined later during limb development. In this layout of growth-stimulating signals, BMPs appear as differentiation-promoting agents, antagonizing signals from the AER and ZPA [Bénazet and Zeller, 2009]. BMPs are not only required for the condensation and bone-forming structures, but their modulated expression levels also determine their identity [Dahn and Fallon, 2000]. The formation of an extra phalange at digit 1 , seeming a transformation to digit 2 , is consistent with a gain of function of BMP as it could be inferred from manipulation of structures in vivo and the high proportion of unrestricted BMP signaling in FBN1deficient cells [Dahn and Fallon, 2000; Nistala et al., 2010]. How this particular FBN1 mutation causes this phenotype is unknown. The EGF_CA module affected in our patient, particularly rich in glutamine, lies in the Cterminal domain likely involved in joining FBN1 molecules [Kuo et al., 2007]. In addition, this region binds the BMP-10 pro-peptide with high affinity $\left(\mathrm{K}_{\mathrm{D}}=30 \mathrm{nM}\right)$ in an in vitro Biacore assay [Sengle et al., 2008], so it could also bind other related factors. In fact, the fusion of carpal bones is also observed in GDF6/BMP13 knockout mice [Settle et al., 2003], suggesting that this EGF_CA module at the C-terminal domain of FBN1 actively participates in the complex finely tuned TGF- $\beta$-related signaling network that participates in multiple developmental processes [Sakai et al., 2016].

The origin of the patient's mutation was also addressed in this study. A report analyzing 1,013 patients with MFS revealed that $48 \%$ did not have a previous family history, either because related carriers were asymptomatic or the
152

Mol Syndromol 2017;8:148-154

DOI: $10.1159 / 000467909$
Martínez-Quintana et al. 
mutation appeared sporadically [Faivre et al., 2007]. One hypothesis for this type of event is mosaicism due to the accumulation of deleterious mutations in the spermatogonia of older fathers [Sakai et al., 2016]. However, maternal mosaicism is demonstrated in 3 cases [Montgomery et al., 1998; Sípek et al., 2014; Rekondo et al., 2016], while paternal mosaicism is reported in only 2 cases [Collod-Béroud et al., 1999; Tekin et al., 2007]. In our study, the father of the proband is a gonosomal mosaic, evidenced by the presence of mutant alleles in blood, buccal epithelium and, based on his daughter's genotype, in sperm. The fraction of mutant alleles (13.8\%) predicts that roughly $25 \%$ of the father's cells carry the mutation, indicating that it arose in the father at a very early postzygotic mitosis. Despite this high level of mosaicism, the father does not show evidence of gross skeletal and cardiac MFS-related symptoms.

Genetic mosaicism is common at the genomic scale [Huang et al., 2014], and its impact in human disease is evident [Biesecker and Spinner, 2013]. While somatic mosaicism arising during development may lead to disease exclusively in the individual where the mutation originated, gonadal (during gametogenesis) or gonosomal mosaicism (affecting both somatic and germ cells) arising during parental development have a high probability of transmitting de novo mutations to the offspring. Therefore, in the absence of evident phenotypic features in parents with a high degree of mosaicism, we would like to stress the importance of detailed parental genetic screening in counseling sporadic cases of MFS, so the risk of recurrence may be evaluated.

\section{Acknowledgments}

We would like to express our gratitude to the patient and family members for participating in this study. We also thank the second reviewer for making a major contribution to the final version of the manuscript. N.C.-S. was a visitor from the undergraduate student corporate training program at the Universidad Miguel Hernández (Elche, Spain). Funding for this study has been provided by the Servicio Canario de Salud.

\section{Statement of Ethics}

The patient and their closest relatives all agreed to participate in this study through written informed consent. Research was ethically conducted in accordance with the World Medical Association Declaration of Helsinki. This study is part of a research program studying the genetics of cardiovascular disease in Gran Canaria, that has been approved by the Ethics Committee of the Complejo Hospitalario Universitario Insular Materno Infantil.

\section{Disclosure Statement}

The authors have no conflicts of interest to declare.

\section{References}

Arbustini E, Grasso M, Ansaldi S, Malattia C, Pilotto A, et al: Identification of sixty-two novel and twelve known FBN1 mutations in eightyone unrelated probands with Marfan syndrome and other fibrillinopathies. Hum $\mathrm{Mu}-$ tat 26:494 (2005).

Bénazet JD, Zeller R: Vertebrate limb development: moving from classical morphogen gradients to an integrated 4-dimensional patterning system. Cold Spring Harb Perspect Biol 1:a001339 (2009).

Biesecker LG, Spinner NB: A genomic view of mosaicism and human disease. Nat Rev Genet 14:307-320 (2013).

Collod-Béroud G, Lackmy-Port-Lys M, Jondeau G, Mathieu M, Maingourd Y, et al: Demonstration of the recurrence of Marfan-like skeletal and cardiovascular manifestations due to germline mosaicism for an FBN1 mutation. Am J Hum Genet 65:917-921 (1999).
Collod-Béroud G, Le Bourdelles S, Ades L, AlaKokko L, Booms P, et al: Update of the UMD$F B N 1$ mutation database and creation of an FBN1 polymorphism database. Hum Mutat 22:199-208 (2003).

Dahn RD, Fallon JF: Interdigital regulation of digit identity and homeotic transformation by modulated BMP signaling. Science 289:438441 (2000).

Dietz HC, Saraiva JM, Pyeritz RE, Cutting GR, Francomano CA: Clustering of fibrillin (FBN1) missense mutations in Marfan syndrome patients at cysteine residues in EGFlike domains. Hum Mutat 1:366-374 (1992).

Faivre L, Collod-Béroud G, Loeys BL, Child A, Binquet C, et al: Effect of mutation type and location on clinical outcome in 1,013 probands with Marfan syndrome or related phenotypes and FBN1 mutations: an international study. Am J Hum Genet 81:454-466 (2007).
Huang AY, Xu X, Ye AY, Wu Q, Yan L, et al: Postzygotic single-nucleotide mosaicisms in whole-genome sequences of clinically unremarkable individuals. Cell Res 24:1311-1327 (2014).

Judge DP, Dietz HC: Marfan's syndrome. Lancet 366:1965-1976 (2005).

Kuo CL, Isogai Z, Keene DR, Hazeki N, Ono RN, et al: Effects of fibrillin-1 degradation on microfibril ultrastructure. J Biol Chem 282: 4007-4020 (2007).

Loeys BL, Dietz HC, Braverman AC, Callewaert BL, De Backer J, et al: The revised Ghent nosology for the Marfan syndrome. J Med Genet 47:476-485 (2010).

Marchler-Bauer A, Derbyshire MK, Gonzales NR, Lu S, Chitsaz F, et al: CDD: NCBI's conserved domain database. Nucleic Acids Res 43:D222-226 (2015).

Martínez-Quintana E, Rodríguez-González F, Garay-Sánchez P, Tugores A: A novel fibrillin 1 gene mutation leading to Marfan syndrome with minimal cardiac features. Mol Syndromol 5:236-240 (2014). 
Montgomery RA, Geraghty MT, Bull E, Gelb BD, Johnson M, et al: Multiple molecular mechanisms underlying subdiagnostic variants of Marfan syndrome. Am J Hum Genet 63: 1703-1711 (1998).

Nistala H, Lee-Arteaga S, Smaldone S, Siciliano G, Carta L, et al: Fibrillin-1 and-2 differentially modulate endogenous TGF- $\beta$ and BMP bioavailability during bone formation. J Cell Biol 190:1107-1121 (2010).

Ng PC, Henikoff S: SIFT: predicting amino acid changes that affect protein function. Nucleic Acids Res 31:3812-3814 (2003).

Rekondo J, Robledo-Inarritu M, Vado Y, Pérez de Nanclares G, Arós F: Marfan syndrome caused by somatic mosaicism in an FBN1 splicing mutation. Rev Esp Cardiol (Engl Edit) 69:520-521 (2016)
Sakai LY, Keene DR, Renard M, De Backer J: FBN1: the disease-causing gene for Marfan syndrome and other genetic disorders. Gene 591:279-291 (2016).

Schrijver I, Liu W, Brenn T, Furthmayr H, Francke U: Cysteine substitutions in epidermal growth factor-like domains of fibrillin-1: distinct effects on biochemical and clinical phenotypes. Am J Hum Genet 65:1007-1020 (1999).

Sengle G, Charbonneau NL, Ono RN, Sasaki T, Alvarez J, et al: Targeting of bone morphogenetic protein growth factor complexes to fibrillin. J Biol Chem 283:13874-13888 (2008).

Settle SH Jr, Rountree RB, Sinha A, Thacker A, Higgins K, Kingsley DM: Multiple joint and skeletal patterning defects caused by single and double mutations in the mouse Gdf6 and Gdf5 genes. Dev Biol 254:116-130 (2003).

Sípek A Jr, Grodecká L, Baxová A, Cibulková P, Dvořáková $\mathrm{M}$, et al: Novel FBN1 gene mutation and maternal germinal mosaicism as the cause of neonatal form of Marfan syndrome. Am J Med Genet A 164A:1559-1564 (2014).
Sunyaev S, Ramensky V, Koch I, Lathe W 3rd, Kondrashov AS, Bork P: Prediction of deleterious human alleles. Hum Mol Genet 10:591597 (2001).

Tekin M, Cengiz FB, Ayberkin E, Kendirli T, Fitoz $S$, et al: Familial neonatal Marfan syndrome due to parental mosaicism of a missense mutation in the FBN1 gene. Am J Med Genet A 143A:875-880 (2007).

Verstraeten A, Alaerts M, Van Laer L, Loeys B: Marfan syndrome and related disorders: 25 years of gene discovery. Hum Mutat 37:524531 (2016).

Walker AH, Najarian D, White DL, Jaffe JF, Kanetsky PA, Rebbeck TR: Collection of genomic DNA by buccal swabs for polymerase chain reaction-based biomarker assays. Environ Health Perspect 107:517-520 (1999). 